\title{
TREX1 polymorphisms associated with autoantibodies in patients with systemic lupus erythematosus
}

\author{
Jin-Wuk Hur • Yoon-Kyoung Sung • Hyoung Doo Shin • \\ Byung Lae Park $\cdot$ Hyun Sub Cheong $\cdot$ Sang-Cheol Bae
}

Published online: 4 April 2008

(C) Springer-Verlag 2008

\section{Erratum to: Rheumatol Int}

\section{DOI 10.1007/s00296-007-0509-0}

The information of TREX1 and TREX2 has been changed in NCBI data base (http://www.ncbi.nlm.nih.gov). Therefore, we would like to update the gene and SNP nomenclatures as in following Table 1 and Fig. 1. Our conclusion should be revised as "ATRIP polymorphism $-455 G>C$ showed protective effect on the production of anti-Ro $\mathrm{Ab}$, and TREX $1+696 C>T$ in exon 1 and ATRIP-TREX1 ht2 [G-T$\mathrm{C}-\mathrm{T}]$ showed also protective effect on the production of anti-dsDNA Ab."

The online version of the original article can be found under doi:10.1007/s00296-007-0509-0.

\section{J.-W. Hur}

Division of Rheumatology, Department of Internal Medicine,

Eulji University College of Medicine,

Seoul 139-711, Republic of Korea

Y.-K. Sung · S.-C. Bae $(\bowtie)$

Department of Internal Medicine, The Hospital for Rheumatic Diseases, Hanyang University Medical Center, 17 Haengdang-Dong, Seongdong-Gu, Seoul 133-792, Republic of Korea

e-mail: scbae@ hanyang.ac.kr

H. D. Shin · B. L. Park · H. S. Cheong Department of Genetic Epidemiology, SNP Genetics, Inc, Seoul 153-803, Republic of Korea 
Table 1 Updated information of genetic polymorphisms

ATRIP ATR (ataxia-

telangiectasia and RAD3-

related)-interacting protein

(MIM \# 606605), TREX1

3-prime@ @epair exonuclease 1

(MIM \# 606609), UCHL5IP

$\mathrm{UCH} 37$-interacting protein 1

(MIM \# 300540), TREX2

3-prime @ repair exonuclease 1

(MIM \# 300370)

\begin{tabular}{|c|c|c|c|c|c|}
\hline \multicolumn{3}{|c|}{ Old information } & \multicolumn{3}{|c|}{ Updated information } \\
\hline Gene & Name & Location & Gene & Name & Location \\
\hline \multirow[t]{12}{*}{ TREXI } & $-21282 G>C$ & Promoter & ATRIP & $-1477 G>C$ & Promoter \\
\hline & $-21074 A>G$ & Promoter & & $-1269 A>G$ & Promoter \\
\hline & $-20717 G>A$ & Promoter & & $-912 G>A$ & Promoter \\
\hline & $-20702 A>C$ & Promoter & & $-897 A>C$ & Promoter \\
\hline & $-20260 G>C$ & Promoter & & $-455 G>C$ & Promoter \\
\hline & $-20144 G>A$ & Promoter & & $-339 G>A$ & Promoter \\
\hline & $-19863 G>C$ & Promoter & & $-58 G>C$ & Promoter \\
\hline & $-2753 A>G$ & Intron & & $17053 A>G$ & Intron9 \\
\hline & $-890 C>A$ & $5^{\prime}$ utr & TREXI & $-725 C>A$ & $5^{\prime}$ utr \\
\hline & $-389 T>C$ & $5^{\prime}$ utr & & $-223 T>C$ & $5^{\prime}$ utr \\
\hline & $-381 C>T$ & $5^{\prime}$ utr & & $-215 C>T$ & $5^{\prime}$ utr \\
\hline & $531 C>T$, Tyr177Tyr & Exon 16 & & $+696 C>T, Y 232 Y$ & Exon1 \\
\hline \multirow[t]{4}{*}{ TREX2 } & $-23386 G>C$ & Promoter & UCHL5IP & $-641 G>C$ & Promoter \\
\hline & $-14703 G>A$ & Intron4 & & $+8043 G>A$ & Intron4 \\
\hline & $-7279 C>T$ & Intron8 & & $+15467 C>T$ & Intron8 \\
\hline & $+1747 C>T$ & Exon1 (UTR) & TREX2 & $-670 C>T$ & Exon1 (UTR) \\
\hline
\end{tabular}

A2. LDs among ATRIP /TREX1 polymorphisms

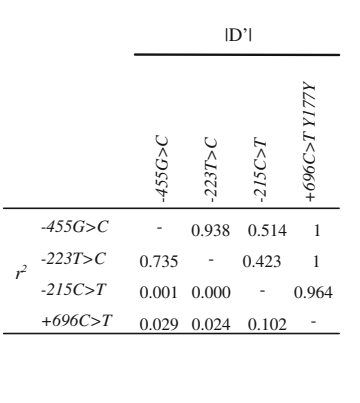

B2. LDs among UCHL5IP /TREX2 polymorphisms

B3. Haplotypes of $U C H L 5 I P$ /TREX2 polymorphisms

A3. Haplotypes of ATRIP /TREX1 polymorphisms

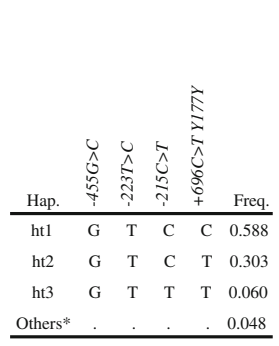

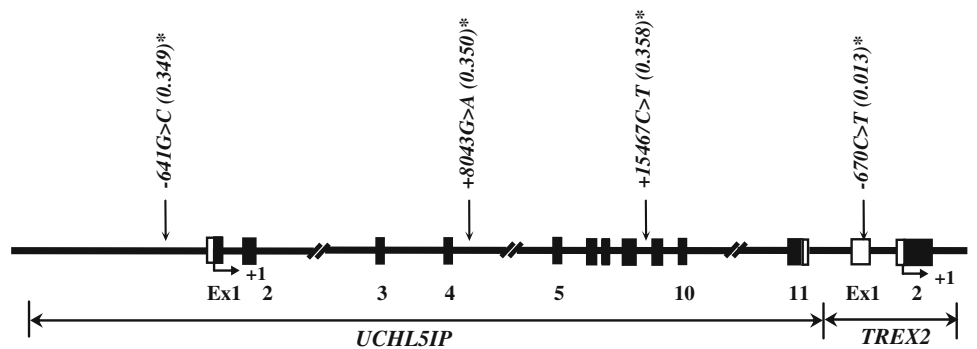
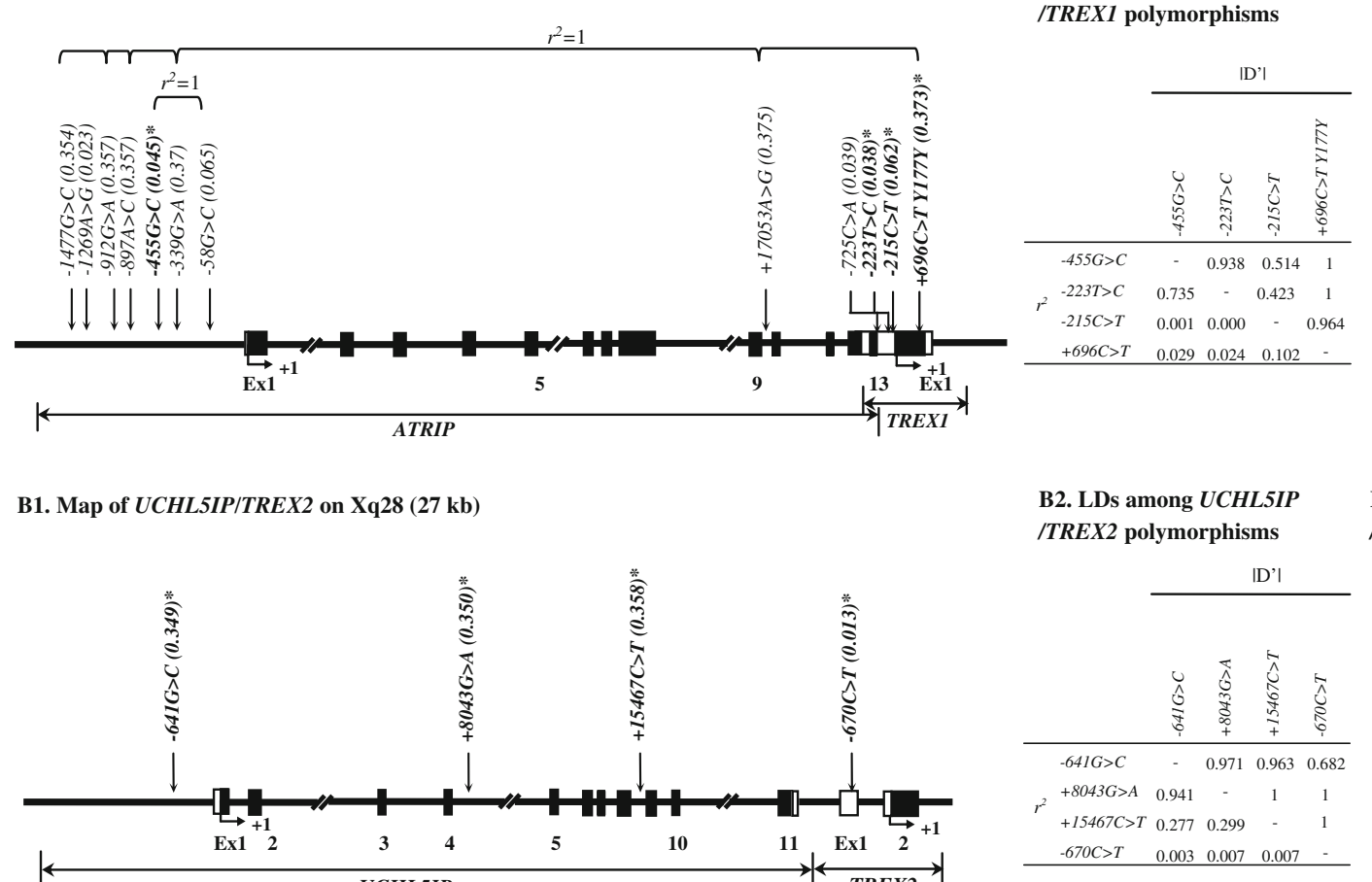

Fig. 1 Gene map and haplotypes of ATRIP/TREX1 and UCHL5IP/ TREX2 genes. Coding exons are marked by black blocks, and $5^{\prime}$ and $3^{\prime}$ UTRs by white blocks. The first base of the translational start site is denoted as nucleotide +1 . Asterisks indicate polymorphisms genotyped in a larger population $(n=1,053)$. The frequencies of polymorphisms not subjected to larger scale genotyping were based on sequence data $(n=24)$. A1 Polymorphisms of ATRIP/TREX1 identified on chromosome 3p21.3-p21.2. A2. LD coefficients $\left(\left|D^{\prime}\right|\right.$ and $\left.r^{2}\right)$ among ATRIP/ TREX1 polymorphisms. A3 Haplotypes of ATRIP/TREX1 exhibiting frequencies $\geq 0.05$. Others asterisks contain rare haplotypes: CCCC, CTCC, GCCC, and CCTC. B1 Polymorphisms of UCHL5IP/TREX2

identified on chromosome Xq28. B2. LD coefficients $\left(\left|D^{\prime}\right|\right.$ and $\left.r^{2}\right)$ among UCHL5IP/TREX2 polymorphisms. B3 Haplotypes of $U C H L 5 I P / T R E X 2$ exhibiting frequencies $\geq 0.05$. Others double asterisks contain rare haplotypes: GGCT, GACC, CGTC, CGCT, and CGCC. Reference sequences of ATRIP/TREX1 was based on the genomic contig, NT_022517 and mRNA transcripts, NM_130384 and NM_016381 for ATRIP and TREX1, respectively. Reference sequences of UCHL5IP/TREX2 was based on the genomic contig, NT_011726 and mRNA transcripts, NM_207107 and NM_080701 for UCHL5IP and TREX2, respectively 